\title{
Low Energy BSE Imaging with a New Scintillation Detector
}

\author{
J. Kološová ${ }^{1}$, J. Jiruše ${ }^{1}$, J. Fiala ${ }^{1}$ and J. Beránek ${ }^{1}$ \\ ${ }^{1}$ TESCAN Brno, s.r.o., Libusina trida 1, Brno, Czech Republic.
}

More and more applications of the scanning electron microscope (SEM) rely on a low electron energy because it decreases the depth of specimen radiation damage. It also enables a clear visualization of nonconductive samples, surface structures are better resolved, and new types of contrast can be observed $[1,2]$. In the case of backscattered electron (BSE) imaging, working at low primary beam energies (and currents) puts high demands on the actual detector design because of the weak signal intensities. The sensitivity of the most commonly used scintillation detectors drops rapidly in the region of energies under $3 \mathrm{keV}$.

We have recently developed a new scintillation type BSE detector with enhanced sensitivity in the low energy region [3]. Thanks to a special surface treatment the dead layers on the detection surface were reduced and the detection limit of the detector was pushed down to $200 \mathrm{eV}$, see Figure 1 . The good collection efficiency together with the high light yield and short decay time of the scintillation material guarantee high signal to noise ratio even at high scanning rates and low probe currents. This is crucial for many applications, like for example 3D analysis of radiation sensitive or non-conductive samples.

The benefits of this BSE detector can also be exploited in the semiconductor industry, especially in the field of failure analysis. The fast 3D reconstruction using the new BSE detector at low primary energies gives contrast which offers a better depth resolution. The signal contains information only from the top layers and is not affected by the signal from the bulk, see Figures $2 \mathrm{~A}$ and $2 \mathrm{~B}$.

In the surface-enhanced Raman microscopy (SERS) the molecules to be analyzed are adsorbed on rough surfaces, such as partially metal-coated plasma etched polystyrene spheres. This substrate is nonconductive, thus correlative SEM and Raman imaging [4] requires low primary energies and very low probe currents, see Figure 2C.

Another application is the 3D reconstruction of resin-embedded biological samples where low primary beam energies and currents are necessary to minimize possible radiation damage and sample charging. Because of large quantities of analyzed material, high imaging speeds are required, i.e. FIB-milling plus milled surface imaging must fit within the limit of 2 minutes, and pixel resolution of the image should be higher than $10 \mathrm{~nm}$ per pixel, see Figure 3.

\section{References:}

[1] DC Bell, N Erdman in "Low Voltage Electron microscopy: Principles and Applications", ed. DC Bell and N Erdman, (John Wiley \& Sons, Chichester) p. 1.

[2] L Frank et al, Materials 5 (2012), p. 2731.

[3] J Kolosova et al, Proceedings of the $18^{\text {th }}$ International Microscopy Congress (2014).

[4] J Jiruse et al, Microscopy and Microanalysis 20 (2014), p. 990. 

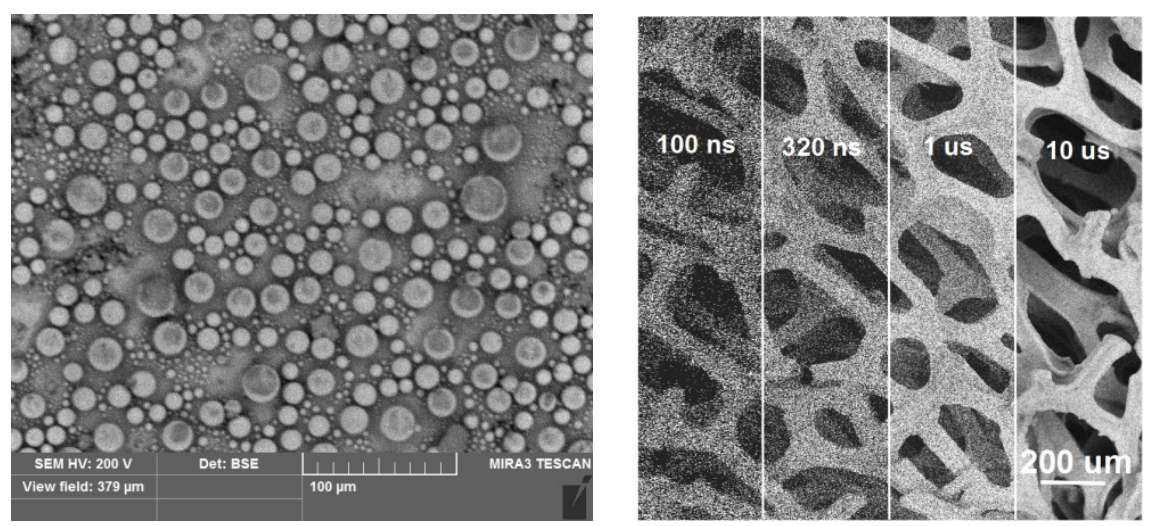

Figure 1. Detection limits of the new BSE detector. A): Tin on carbon sample imaged at $200 \mathrm{eV}$ primary beam energy and $65 \mathrm{pA}$ probe current. B): Ni grid imaged at various pixel dwell times (from $320 \mathrm{~ns}$ to $32 \mu \mathrm{s}$ ), $500 \mathrm{eV}$ primary energy and $500 \mathrm{pA}$ beam current. Imaging at high scanning rates in the low energy region is possible.
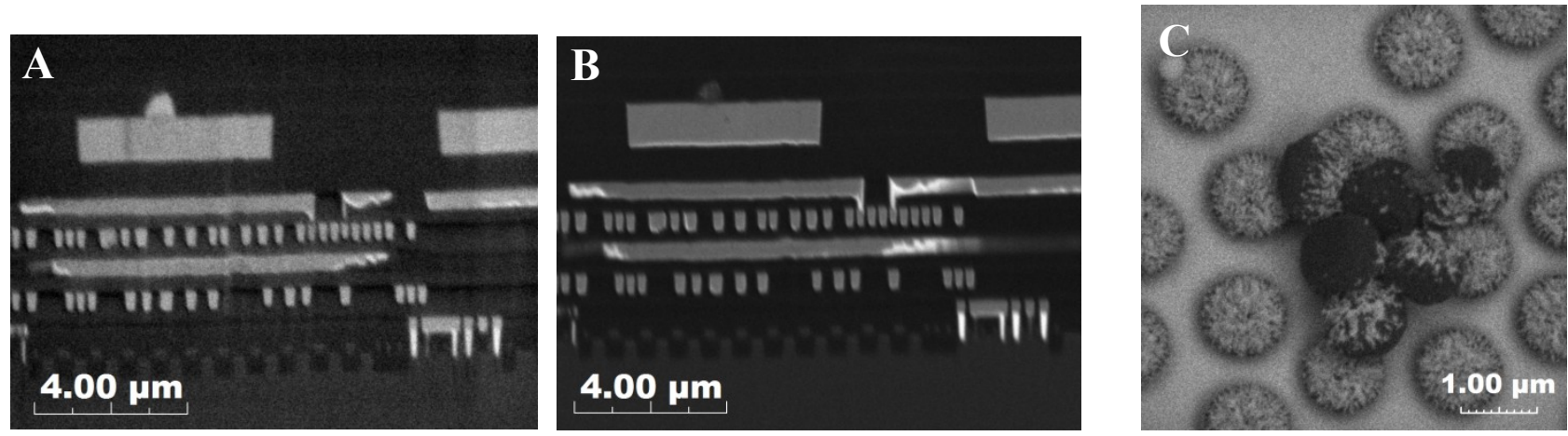

Figure 2. IC cross section imaged at A) $1 \mathrm{keV}$ and B) $5 \mathrm{keV}$ primary energies showing enhanced surface sensitivity at lower primary beam energies. C): Au-coated plasma-etched polystyrene spheres (SERS substrate). Image was taken at $1 \mathrm{keV}$ primary energy and $45 \mathrm{pA}$ probe current.
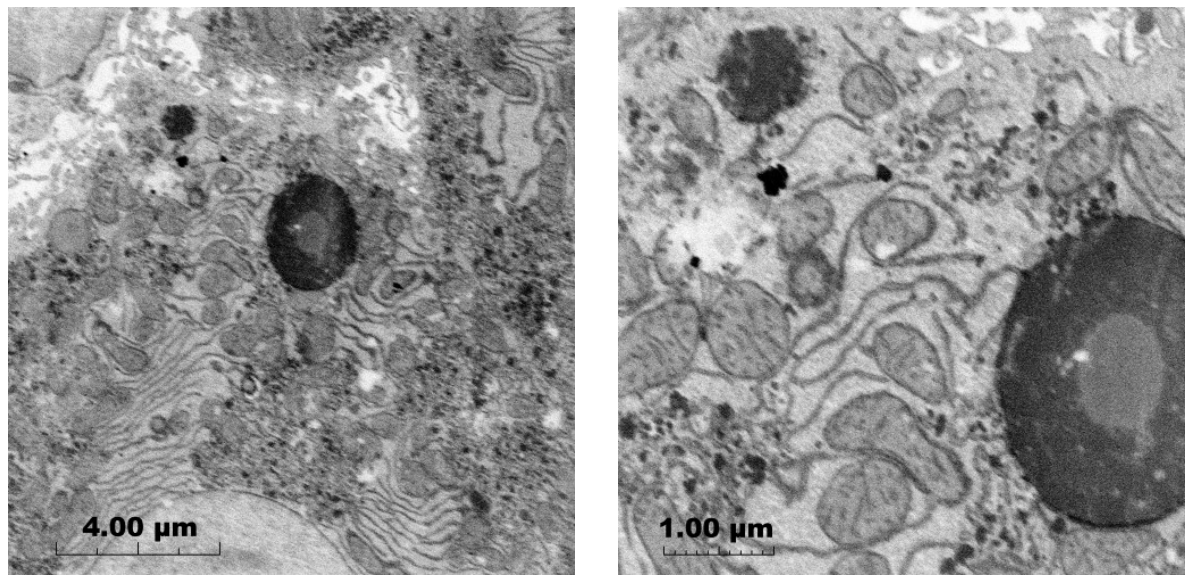

Figure 3. Stained liver section imaged at $2 \mathrm{keV}$ primary energy and $100 \mathrm{pA}$ probe current. Acquisition times of the images ( 2048 x 2048 pxl) were 49 seconds. Imaging was done with the low energy BSE detector placed inside the column. Sample courtesy Gatan. 